九州大学学術情報リポジトリ

Kyushu University Institutional Repository

\title{
LOCALLY MOST POWERFUL RANK TESTS FOR INDEPENDENCE
}

Shirahata, Shingo

Faculty of Engineering, Osaka University

https://doi.org/10.5109/13079

出版情報：統計数理研究. 16 (1/2)，pp.11-21，1974-03. Research Association of Statistical Sciences バージョン :

権利関係 : 


\title{
LOCALLY MOST POWERFUL RANK TESTS FOR INDEPENDENCE
}

\author{
By \\ Shingo Shirahata* \\ (Received December 1, 1972)
}

\section{Introduction and summary.}

In testing independence of two random variables based on rank statistics, several rank statistics such as Spearman's $\rho$, Kendall's $\tau$, normal score statistics, etc. are available and performance of the tests based on these statistics has been studied for some models; see, e. g., Bhuchongkul [3], Farlie [5], Hájek and Šidák [8] and Konijn [9].

In this paper we study the one-sided and two-sided locally most powerful rank tests (LMPRT) to test the independence of $p$-dimensional random variables $(p \geqq 2)$ with $q$-parameters, where the independence is characterized by the value zero for all parameters. The term 'locally' means that parameters are included in some neighbourhood of the origin. Two-sided LMPRT will be considered only when onesided LMPRT does not exist.

In Sections 5 and 6 asymptotic normality of the test statistic in the one-sided LMPRT will be studied.

In this paper, only total independency is adopted as a null hypothesis, so that neither pairwise independence nor general independency of sets of variables will not be dealt with. These two independencies have been studied in Puri and Sen [12] and their other several papers and also in Anderson [2] for the normal case.

\section{Notations and assumptions.}

Let $\boldsymbol{X}_{N 1}, \cdots, \boldsymbol{X}_{N N}$ be mutually independent random variables and each $\boldsymbol{X}_{N i}=$ $\left(X_{N i 1}, \cdots, X_{N i p}\right)^{\prime}$ be distributed with a density function $f\left(x_{1}, \cdots, x_{p}, c_{N i 1} \theta_{1}, \cdots, c_{N i q} \theta_{q}\right)$, where the function form of $f$ is known, all c's are known constants and $\theta$ 's are parameters each of which has a range containing the origin. We assume that, if and only if all $\theta$ 's are zero, there exist some density functions $f_{1}, \cdots, f_{p}$ such that $f\left(x_{1}, \cdots, x_{p}, 0, \cdots, 0\right)=\prod_{i=1}^{p} f_{i}\left(x_{i}\right)$.

To simplify the notations, we shall write $f\left(x_{1}, \cdots, x_{p}, c_{N i_{1}} \theta_{1}, \cdots, c_{N i q} \theta_{q}\right), f\left(x_{1}, \cdots\right.$, $\left.x_{p}, \theta_{1}, \cdots, \theta_{q}\right)$ and $f\left(x_{1}, \cdots, x_{p}, 0, \cdots, 0\right)$ as $f\left(\boldsymbol{x}, \boldsymbol{c}_{N i} \theta\right), f(\boldsymbol{x}, \theta)$ and $f(\boldsymbol{x})$ respectively.

Now we give some notations and assumptions to be used throughout the paper.

* Faculty of Engineering Science, Osaka University, Toyonaka, Osaka. 
First,

$$
\begin{gathered}
\phi_{r}(\boldsymbol{x}, \theta)=\left(\partial / \partial \theta_{r}\right) \log f(\boldsymbol{x}, \theta), \\
\psi_{r}(\boldsymbol{x})=\phi_{r}(\boldsymbol{x}, \mathbf{0}), \\
\psi_{r s}(\boldsymbol{x}, \theta)=\left\{\left(\partial^{2} / \partial \theta_{r} \partial \theta_{s}\right) f(\boldsymbol{x}, \theta)\right\} / f(\boldsymbol{x}, \theta), \\
\phi_{r s}(\boldsymbol{x})=\phi_{r s}(\boldsymbol{x}, \mathbf{0}),
\end{gathered}
$$

for $r, s=1, \cdots, q$ and $\mathbf{0}=(0, \cdots, 0)$. Next,

Assumption $\mathrm{A}_{1} \cdot f(\boldsymbol{x}, \theta)$ is totally differentiable with respect to $\theta$ in some open set which contains the origin of $R^{q}$, and

$$
E_{\theta}\left|\psi_{r}(\boldsymbol{X}, \theta)\right| \longrightarrow E_{0}\left|\psi_{r}(\boldsymbol{X})\right| \quad \text { as }|\theta| \rightarrow 0 \text { for } r=1, \cdots, q,
$$

where the expectation $E_{\theta}$ is computed under the distribution with the density $f(\boldsymbol{x}, \theta)$.

Assumption $\mathrm{A}_{2}$. Constants $c_{N i r}, i=1, \cdots, N, r=1, \cdots, q$, are known such that some of them are not equal to zero for every $N$.

Assumption $\mathrm{A}_{3}$ (One-sided hypothesis). $\sum_{r=1}^{q} \theta_{r}$ is positive, and

$$
\begin{gathered}
\theta_{r} \longrightarrow 0 \quad \text { for } r=1, \cdots, q, \\
\theta_{r}\left(\sum_{i=1}^{q} \theta_{i}\right)^{-1} \longrightarrow \lambda_{r} \quad \text { for } r=1, \cdots, q,
\end{gathered}
$$

where $\lambda_{1}, \cdots, \lambda_{q}$ are fixed numbers.

In the one-parameter case, Assumption $A_{3}$ reduces to the ordinary one-sided hypothesis. Similary the following $\mathrm{A}_{3}{ }_{3}$ reduces to the ordinary two-sided hypothesis.

Assumption $\mathrm{A}^{\prime}{ }_{3}$ (Two-sided hypothesis). $\sum_{r=1}^{q} \theta_{r}$ is not equal to zero, and the limiting conditions (2.6) and (2.7) hold.

AsSumption $\mathrm{A}_{4}$. $\left(\partial / \partial \theta_{r}\right) f(\boldsymbol{x}, \theta)$ is totally differentiable with respect to $\theta$ in some open set which contains the origin of $R^{q}$ for $r=1, \cdots, q$, and

$$
E_{\theta}\left|\phi_{r s}(\boldsymbol{X}, \theta)\right| \longrightarrow E_{0}\left|\phi_{r s}(\boldsymbol{X})\right| \quad \text { as }|\theta| \rightarrow 0 \text { for } r, s=1, \cdots, q .
$$

This Assumption $\mathrm{A}_{4}$ will be used to derive two-sided LMPRT. Finally we state the hypotheses involved.

$$
\begin{aligned}
& H_{N 0}: \text { Each } \boldsymbol{X}_{N i} \text { has a density function } f(\boldsymbol{x}) . \\
& H_{N \boldsymbol{c} \theta}: \text { Each } \boldsymbol{X}_{N i} \text { has a density function } f\left(\boldsymbol{x}, \boldsymbol{c}_{N i} \theta\right) . \\
& H_{N \theta}: \text { Each } \boldsymbol{X}_{N i} \text { has a density function } f(\boldsymbol{x}, \theta) .
\end{aligned}
$$

\section{Locally most powerful rank tests.}

Let $\boldsymbol{R}_{N}=\left(\boldsymbol{R}_{N 1}, \cdots, \boldsymbol{R}_{N N}\right)$ be the rank matrix of $\boldsymbol{X}=\left(\boldsymbol{X}_{N_{1}}, \cdots, \boldsymbol{X}_{N N}\right)$, where $\boldsymbol{R}_{N i}=$ $\left(R_{N i 1}, \cdots, R_{N i p}\right)^{\prime}$ and $R_{N i r}$ denotes the rank of $X_{N i r}$ among $X_{N i r}, \cdots, X_{N N r}$. Every test considered in this paper is based on a function of $\boldsymbol{R}_{N}$. First, we state the following theorem.

TheOREM 1. Under Assumptions $A_{1}, A_{2}$ and $A_{3}$ the test for $H_{N_{0}}$ against $H_{N c \theta}$ 
based on the statistic $S_{N}\left(\boldsymbol{R}_{N}\right)$, where

$$
S_{N}\left(\boldsymbol{R}_{N}\right)=\sum_{k=1}^{N} \sum_{r=1}^{q} c_{N k r} \lambda_{r} E_{0}\left[\psi_{r}\left(\boldsymbol{X}_{N k}\right) \mid \boldsymbol{R}_{N k}\right],
$$

that is $H_{N 0}$, is rejected if $S_{N}\left(\boldsymbol{R}_{N}\right)$ is larger than a given constant and accepted otherwise, is locally most powerful among rank tests at the respective level.

Proof. The proof goes along the same line as in [8], Chapter II. Let $P_{\theta}(A)$ be the probability of an event $A$ under $H_{N c \theta}$. Let $\boldsymbol{r}=\left(\boldsymbol{r}_{1}, \cdots, \boldsymbol{r}_{N}\right)$ be the observed rank matrix. Then we have

$$
\begin{aligned}
& P_{\theta}\left(\boldsymbol{R}_{N}=\boldsymbol{r}\right)-P_{0}\left(\boldsymbol{R}_{N}=\boldsymbol{r}\right) \\
& =\int_{\boldsymbol{R}_{N}=r} \cdots \int_{i=1}\left(\prod_{i=1}^{N} f\left(\boldsymbol{x}_{i}, \boldsymbol{c}_{N i} \theta\right)-\prod_{i=1}^{N} f\left(\boldsymbol{x}_{i}\right)\right) d \boldsymbol{x}_{1} \cdots d \boldsymbol{x}_{N} \\
& =\sum_{k=1}^{N} \sum_{r=1}^{q} c_{N k r} \theta_{r} \int_{\boldsymbol{R}_{N}=r} \cdots \int\left[\prod_{i=1}^{k-1} f\left(\boldsymbol{x}_{i}, \boldsymbol{c}_{N i} \theta\right) \prod_{i=k}^{N} f\left(\boldsymbol{x}_{i}\right)\right]\left(c_{N k r} \theta_{r} f\left(\boldsymbol{x}_{k}\right)\right)^{-1} \\
& \times\left[f\left(\boldsymbol{x}_{k}, c_{N k 1} \theta_{1}, \cdots, c_{N k r} \theta_{r}, 0, \cdots, 0\right)\right. \\
& \left.-f\left(\boldsymbol{x}_{k}, c_{N k 1} \theta_{1}, \cdots, c_{N k r-1} \theta_{r-1}, 0, \cdots, 0\right)\right] d \boldsymbol{x}_{1} \cdots d \boldsymbol{x}_{N} .
\end{aligned}
$$

For simplicity, let us denote by $F$ the function under the integral sign of (3.4). Then due to the first half of $A_{1}$,

$$
F \longrightarrow \phi_{r}\left(\boldsymbol{x}_{k}\right) \prod_{i=1}^{N} f\left(\boldsymbol{x}_{i}\right) \quad \text { as }|\theta| \rightarrow 0,
$$

and we easily get the following relation.

$$
\begin{aligned}
& \int \cdots \int|F| d \boldsymbol{x}_{1} \cdots d \boldsymbol{x}_{N} \\
& \leqq\left(c_{N k r} \theta_{r}\right)^{-1} \int_{0}^{c_{N k r} \theta_{r}} \int \cdots \int\left|\frac{\partial}{\partial t} f\left(\boldsymbol{x}, c_{N k 1} \theta_{1}, \cdots, c_{N k r-1} \theta_{r-1}, t, 0, \cdots, 0\right)\right| d \boldsymbol{x} d t .
\end{aligned}
$$

Using $A_{1}$, the right side of (3.6) converges to

$$
\int \cdots \int\left|\frac{\partial}{\partial \theta_{r}} f(\boldsymbol{x}, \mathbf{0})\right| d \boldsymbol{x}=\int \cdots \int_{R^{p N}}\left|\phi_{r}\left(\boldsymbol{x}_{k}\right)\right| \prod_{i=1}^{N} f\left(\boldsymbol{x}_{i}\right) d \boldsymbol{x}_{1} \cdots d \boldsymbol{x}_{N} .
$$

On combining the facts (3.5), (3.6) and (3.7), from Theorem II 4.2 of [8], we get

$$
\int_{\boldsymbol{R}_{N}=r} \cdots \int_{\boldsymbol{R}_{N}=r} F d \boldsymbol{x}_{1} \cdots d \boldsymbol{x}_{N} \longrightarrow \int_{r} \cdots \phi_{r}\left(\boldsymbol{x}_{k}\right) \prod_{i=1}^{N} f\left(\boldsymbol{x}_{i}\right) d \boldsymbol{x}_{1} \cdots d \boldsymbol{x}_{N} \quad \text { as }|\theta| \rightarrow 0 .
$$

Therefore,

$$
\begin{aligned}
& \left(P_{\theta}\left(\boldsymbol{R}_{N}=\boldsymbol{r}\right)-P_{0}\left(\boldsymbol{R}_{N}=\boldsymbol{r}\right)\right)\left(\sum_{r=1}^{q} \theta_{r}\right)^{-1} \\
& \longrightarrow \sum_{k=1}^{N} \sum_{r=1}^{q} c_{N k r} \lambda_{r} \int_{\boldsymbol{R}_{N}=r} \cdots \int \phi_{r}\left(\boldsymbol{x}_{k}\right) \prod_{i=1}^{N} f\left(\boldsymbol{x}_{i}\right) d \boldsymbol{x}_{1} \cdots d \boldsymbol{x}_{N} \\
& =(N !)^{-p} \sum_{k=1}^{N} \sum_{r=1}^{q} c_{N k r} \lambda_{r} E_{0}\left[\psi_{r}\left(\boldsymbol{X}_{N k}\right) \mid \boldsymbol{R}_{N_{k}}=\boldsymbol{r}_{k}\right]
\end{aligned}
$$




$$
=(N !)^{-p} S_{N}\left(\boldsymbol{R}_{N}=\boldsymbol{r}\right),
$$

where the limit was taken subject to $A_{3}$. This fact implies that there exists an $\varepsilon>0$ such that if $0<\sum_{r=1}^{q} \theta_{r}<\varepsilon,\left|\theta_{r}\right|<\varepsilon$ and $\left|\theta_{r}\left(\sum_{i=1}^{q} \theta_{i}\right)^{-1}-\lambda_{r}\right|<\varepsilon$ for $r=1, \cdots, q$, then,

$$
S_{N}\left(\boldsymbol{R}_{N}=\boldsymbol{r}\right)>S_{N}\left(\boldsymbol{R}_{N}=\boldsymbol{r}^{\prime}\right) \Leftrightarrow P_{\theta}\left(\boldsymbol{R}_{N}=\boldsymbol{r}\right)>P_{\theta}\left(\boldsymbol{R}_{N}=\boldsymbol{r}^{\prime}\right) .
$$

In view of Neyman-Pearson lemma, this completes the proof.

In some problems of testing independence, all c's are equal and $S_{N}\left(\boldsymbol{R}_{N}\right)$ turns out to be trivial or identically equal to zero (cf. Example (d) in Section 4). In such cases we consider the two-sided case under the following assumption.

Assumption $A_{5}$.

$$
\sum_{k=1}^{N} E_{0}\left(\psi_{r}\left(\boldsymbol{X}_{N k}\right) \mid \boldsymbol{R}_{N k}\right)=0 \quad \text { for } r=1, \cdots, q .
$$

Two-sided LMPRT is given by the following theorem.

Theorem 2. Assume Assumptions $A_{1}, A^{\prime}{ }_{3}, A_{4}$ and $A_{5}$ and also that c's are equal to 1. Then locally most powerful rank test for $H_{N 0}$ against $H_{N \theta}$ is based on the statistic $T_{N}\left(\boldsymbol{R}_{N}\right)$,

$$
\begin{aligned}
& T_{N}\left(\boldsymbol{R}_{N}\right)=\sum_{k=1}^{N} \sum_{r=1}^{q} \sum_{s=1}^{q} \lambda_{r} \lambda_{s} E_{0}\left[\psi_{r s}\left(\boldsymbol{X}_{N k}\right) \mid \boldsymbol{R}_{N k}\right] \\
& +\sum_{k \neq k^{\prime}} \sum_{r=1}^{q} \sum_{s=1}^{q} \lambda_{r} \lambda_{s} E_{0}\left[\psi_{r}\left(\boldsymbol{X}_{N k}\right) \psi_{s}\left(\boldsymbol{X}_{N k^{\prime}}\right) \mid \boldsymbol{R}_{N k}, \boldsymbol{R}_{N k^{\prime}}\right] .
\end{aligned}
$$

Proof. As in the proof of Theorem 1, we easily find that

$$
\begin{aligned}
& P_{\theta}\left(\boldsymbol{R}_{N}=\boldsymbol{r}\right)-P_{0}\left(\boldsymbol{R}_{N}=\boldsymbol{r}\right) \\
& =\sum_{k=1}^{N} \sum_{r=1}^{q} \int_{\boldsymbol{R}_{N}=r} \cdots \int_{i=1}^{k-1} f\left(\boldsymbol{x}_{i}, \theta\right) \prod_{i=k+1}^{N} f\left(\boldsymbol{x}_{i}\right) \int_{0}^{\theta_{r}} \frac{\partial}{\partial t} f\left(\boldsymbol{x}_{k}, \theta_{1}, \cdots, \theta_{r-1}, t, 0, \cdots, 0\right) d t d \boldsymbol{x}_{1} \cdots d \boldsymbol{x}_{N} \\
& =\sum_{k=1}^{N} \sum_{r=1}^{q} \int_{\boldsymbol{R}_{N}=r} \ldots \int_{i=1}^{k-1} f\left(\boldsymbol{x}_{i}, \theta\right) \prod_{i=k+1}^{N} f\left(\boldsymbol{x}_{i}\right) \int_{0}^{\theta_{r}} \int_{0}^{t} \frac{\partial^{2}}{\partial s^{2}} f\left(\boldsymbol{x}_{k}, \theta_{1}, \cdots, \theta_{r-1}, s, 0, \cdots, 0\right) d s d t d \boldsymbol{x}_{1} \cdots d \boldsymbol{x}_{N} \\
& +\sum_{k=1}^{N} \sum_{r=1}^{q} \sum_{s=1}^{r-1} \theta_{r} \int_{\boldsymbol{R}_{N}=r} \cdots \int_{i=1}^{k-1} f\left(\boldsymbol{x}_{i}, \theta\right) \prod_{i=k+1}^{N} f\left(\boldsymbol{x}_{i}\right) \int_{0}^{\theta_{s}} \frac{\partial^{2}}{\partial \theta_{r} \partial t} f\left(\boldsymbol{x}_{k}, \theta_{1}, \cdots, \theta_{s-1}, t, 0, \cdots, 0\right) \\
& +\sum_{k=1}^{N} \sum_{r=1}^{q} \theta_{r} \int_{\boldsymbol{R}_{N}=r} \ldots \int_{\frac{r}{\partial \theta_{r}}} \frac{\partial}{\partial t d \boldsymbol{x}_{1} \cdots d \boldsymbol{x}_{N}}
\end{aligned}
$$

Let us denote (3.16), (3.17) and (3.18) by $B_{1}, B_{2}$ and $B_{3}$ respectively and consider

$$
\left(P_{\theta}\left(\boldsymbol{R}_{N}=\boldsymbol{r}\right)-P_{0}\left(\boldsymbol{R}_{N}=\boldsymbol{r}\right)\right)\left(\sum_{r=1}^{q} \theta_{r}\right)^{-2},
$$

when $|\theta| \rightarrow 0$ subject to $\mathrm{A}_{3}^{\prime}$.

First it can be easily shown, as in the proof of Theorem 1 , by $A_{4}$ and Theorem II. 4.2 of [8], that $B_{1}\left(\sum_{r=1}^{q} \theta_{r}\right)^{-2}$ and $B_{2}\left(\sum_{r=1}^{q} \theta_{r}\right)^{-2}$ tend to 


$$
\begin{aligned}
& \frac{1}{2}(N !)^{-p} \sum_{k=1}^{N} \sum_{r=1}^{q} \lambda_{r}^{2} E_{0}\left[\phi_{r r}\left(\boldsymbol{X}_{N k}\right) \mid \boldsymbol{R}_{N k}=\boldsymbol{r}_{k}\right] \\
& (N !)^{-p} \sum_{k=1}^{N} \sum_{r=1}^{q} \sum_{s=1}^{r-1} \lambda_{r} \lambda_{s} E_{0}\left[\psi_{r s}\left(\boldsymbol{X}_{N k}\right) \mid \boldsymbol{R}_{N k}=\boldsymbol{r}_{k}\right]
\end{aligned}
$$

respectively.

Next we turn to $B_{3}\left(\sum_{r=1}^{q} \theta_{r}\right)^{-2}$, which can be written as

$$
\begin{aligned}
& B_{3}\left(\sum_{i=1}^{q} \theta_{r}\right)^{-2} \\
& =\left(\sum_{i=1}^{q} \theta_{r}\right)^{-2} \sum_{k=1}^{N} \sum_{r=1}^{q} \theta_{r} \int \cdots \int\left[\sum_{\boldsymbol{R}_{N}=r}^{k-1}\left\{\left(f\left(\boldsymbol{x}_{k^{\prime}}, \theta\right)-f\left(\boldsymbol{x}_{k^{\prime}}\right)\right) \prod_{i=1}^{k^{\prime}-1} f\left(\boldsymbol{x}_{i}, \theta\right) \prod_{i=k^{\prime}+1}^{k-1} f\left(\boldsymbol{x}_{i}\right)\right\}\right. \\
& \left.+\prod_{i=1}^{k-1} f\left(\boldsymbol{x}_{i}\right)\right] \times \frac{\partial}{\partial \theta_{r}} f\left(\boldsymbol{x}_{k}, \boldsymbol{0}\right) \prod_{i=k+1}^{N} f\left(\boldsymbol{x}_{i}\right) d \boldsymbol{x}_{1} \cdots d \boldsymbol{x}_{N} \\
& =\left(\sum_{i=1}^{q} \theta_{r}\right)^{-2} \sum_{k^{\prime}<k} \sum_{r=1}^{q} \sum_{s=1}^{q} \int \cdots \int\left(\prod_{i=1}^{k_{N}-1} f\left(\boldsymbol{x}_{i}, \theta\right) \prod_{i=k^{\prime}+1}^{N} f\left(\boldsymbol{x}_{i}\right)\right) \\
& \times\left(f\left(\boldsymbol{x}_{k}, \theta_{1}, \cdots, \theta_{s}, 0, \cdots, 0\right)-f\left(\boldsymbol{x}_{k}, \theta_{1}, \cdots, \theta_{s-1}, 0, \cdots, 0\right)\right) \phi_{r}\left(\boldsymbol{x}_{k}\right) d \boldsymbol{x} \cdots d \boldsymbol{x}_{N} \\
& +\left(\sum_{i=1}^{q} \theta_{r}\right)^{-2} \sum_{k=1}^{N} \sum_{r=1}^{q} \int_{\boldsymbol{R}_{N}=r} \cdots \int \phi_{r}\left(\boldsymbol{x}_{k}\right) \prod_{i=1}^{N} f\left(\boldsymbol{x}_{i}\right) d \boldsymbol{x}_{1} \cdots d \boldsymbol{x}_{N} \cdot
\end{aligned}
$$

Assumption $A_{5}$ means that the term (3.24) is equal to zero, while $A_{1}$ and $\mathrm{A}^{\prime}{ }_{3}$ jointly imply that the term (3.23) has the limit given by

$$
\begin{aligned}
& \sum_{k^{\prime}} \sum_{k} \sum_{r=1}^{q} \sum_{s=1}^{q} \lambda_{r} \lambda_{s} \int_{\boldsymbol{R}_{N}=r} \cdots \int_{r}\left(\boldsymbol{x}_{k}\right) \phi_{s}\left(\boldsymbol{x}_{k^{\prime}}\right) \prod_{i=1}^{N} f\left(\boldsymbol{x}_{i}\right) d \boldsymbol{x}_{1} \cdots d \boldsymbol{x}_{N} \\
= & (N !)^{-p} \sum_{\boldsymbol{k}^{\prime}<k} \sum_{r=1}^{q} \sum_{s=1}^{q} \lambda_{r} \lambda_{s} E_{0}\left[\phi_{r}\left(\boldsymbol{X}_{N k}\right) \phi_{s}\left(\boldsymbol{X}_{N k^{\prime}}\right) \mid \boldsymbol{R}_{N k}=\boldsymbol{r}_{k}, \boldsymbol{R}_{N k^{\prime}}=\boldsymbol{r}_{k^{\prime}}\right] .
\end{aligned}
$$

From (3.20), (3.21), (3.26), the symmetricity with respect to $k$ and $k^{\prime}$, and the symmetricity with respect to $r$ and $s$, it follows that the expression (3.19) tends to

$$
\begin{aligned}
& \frac{1}{2}(N !)^{-p} \sum_{k=1}^{N} \sum_{r=1}^{q} \lambda_{r}^{2} E_{0}\left[\phi_{r r}\left(\boldsymbol{X}_{N k}\right) \mid \boldsymbol{R}_{N k}=\boldsymbol{r}_{k}\right] \\
+ & \frac{1}{2}(N !)^{-p} \sum_{k=1}^{N} \sum_{r \neq s} \lambda_{r} \lambda_{s} E_{0}\left[\phi_{r s}\left(\boldsymbol{X}_{N k}\right) \mid \boldsymbol{R}_{N k}=\boldsymbol{r}_{k}\right] \\
+ & \frac{1}{2}(N !)^{-p} \sum_{k \neq k^{\prime}} \sum_{r=1}^{q} \sum_{s=1}^{q} \lambda_{r} \lambda_{s} E_{0}\left[\psi_{r}\left(\boldsymbol{X}_{N k}\right) \psi_{s}\left(\boldsymbol{X}_{N k^{\prime}}\right) \mid \boldsymbol{R}_{N k}=\boldsymbol{r}_{k}, \boldsymbol{R}_{N k^{\prime}}=\boldsymbol{r}_{k^{\prime}}\right] \\
= & \frac{1}{2}(N !)^{-p} T_{N}\left(\boldsymbol{R}_{N}=\boldsymbol{r}\right) .
\end{aligned}
$$

The rest of the proof is same as the proof of Theorem 1.

\section{Examples.}

$S_{N}\left(\boldsymbol{R}_{N}\right)$ and $T_{N}\left(\boldsymbol{R}_{N}\right)$ for some models will be given in this section. The latter is restricted to Hájek's model only. We assume that the required assumptions are 
satisfied. In the bi- or tri-variate case, let $\left(\begin{array}{c}R_{1}, \cdots, R_{N} \\ Q_{1}, \cdots, Q_{N}\end{array}\right)$ and $\left(\begin{array}{c}R_{1}, \cdots, R_{N} \\ Q_{1}, \cdots, Q_{N} \\ S_{1}, \cdots, S_{N}\end{array}\right)$ denote the
rank matrix respectively.

(a) Bivariate normal distribution.

Let $\left(X_{1}, Y_{1}\right), \cdots,\left(X_{N}, Y_{N}\right)$ be mutually independent bivariate normal random variables having common mean vector $\left(\mu_{1}, \mu_{2}\right)$ and the dispersion matrix $\left(\begin{array}{ll}1 & c_{k} \rho \\ c_{k} \rho & 1\end{array}\right)$ for $\left(X_{k}, Y_{k}\right)$, where $\mu_{1}, \mu_{2}$ and $\rho>0$ are unknown parameters.

Then the function $\psi$ in Section 2 is easily calculated to be $\left(x-\mu_{1}\right)\left(y-\mu_{2}\right)$, so we can get

$$
S_{N}\left(\boldsymbol{R}_{N}\right)=\sum_{k=1}^{N} c_{k} E_{0} X_{\left(R_{k}\right)} E_{0} Y_{\left(Q_{k}\right)},
$$

where $E_{0} X_{(i)}=E_{0} Y_{(i)}$ is the expectation of the $i$-th order statistic in the sample of size $N$ from the standardized normal distribution. If $c_{k}=1$ for $k=1, \cdots, N$, then $S_{N}\left(\boldsymbol{R}_{N}\right)$ is the well known normal score test statistic.

(b) Trivariate normal distribution.

Let $\left(X_{k}, Y_{k}, Z_{k}\right) k=1, \cdots, N$ be mutually independent normal random variables having common mean vector $\left(\mu_{1}, \mu_{2}, \mu_{3}\right)$ and the dispersion matrix $\left(\begin{array}{ccc}1 & c_{k 1} \rho_{1} & c_{k 2} \rho_{2} \\ c_{k 1} \rho_{1} & 1 & c_{k 3} \rho_{3} \\ c_{k 2} \rho_{2} & c_{k 3} \rho_{3} & 1\end{array}\right)$ for $\left(X_{k}, Y_{k}, Z_{k}\right)$, where $\sum_{r=1}^{3} \rho_{r}>0, \rho_{r}\left(\sum_{i=1}^{3} \rho_{i}\right)^{-1} \rightarrow \lambda_{r}$ and $\rho_{r} \rightarrow 0$ for $r=1,2,3$. A short calculation shows that

$$
\begin{aligned}
S_{N}\left(\boldsymbol{R}_{N}\right)= & \sum_{k=1}^{N} c_{k 1} \lambda_{1} E_{0} X_{\left(R_{k}\right)} E_{0} Y_{\left(Q_{k}\right)}+\sum_{k=1}^{N} c_{k 2} \lambda_{2} E_{0} X_{\left(R_{k}\right)} E_{0} Z_{\left(S_{k}\right)} \\
& +\sum_{k=1}^{N} c_{k 3} \lambda_{3} E_{0} Y_{\left(Q_{k}\right)} E_{0} Z_{\left(S_{k}\right)} .
\end{aligned}
$$

In (a) and (b), Assumption $A_{1}$ is always satisfied.

(c) Farlie's model.

Farlie [5] proposed the following model:

$$
H(x, y)=F(x) G(y)\{1+\alpha A(F(x)) B(G(y))\}, \quad \alpha \geqq 0,
$$

where $F$ and $G$ are distribution functions, and $A$ and $B$ satisfy some regularity conditions. In this model, without loss of generality, we assume that $F$ and $G$ have density functions $f$ and $g$ respectively, and that $A$ and $B$ are bounded and differentiable, and then we adopt (4.3) with $\alpha$ replaced by $c_{k} \alpha$ as the distribution of $\left(x_{k}, y_{k}\right), k=1, \cdots, N$. Then a simple calculation shows that

$$
\begin{aligned}
S_{N}\left(\boldsymbol{R}_{N}\right) & =\sum_{k=1}^{N} c_{k} E_{0}\left[A\left(F\left(X_{k}\right)\right)+F\left(X_{k}\right) A^{\prime}\left(F\left(X_{k}\right)\right) \mid R_{k}\right] \\
& \times E_{0}\left[B\left(G\left(Y_{k}\right)\right)+G\left(Y_{k}\right) B^{\prime}\left(G\left(Y_{k}\right)\right) \mid Q_{k}\right] .
\end{aligned}
$$

Farlie showed that if $A=1-F, B=1-G$ and $c_{k}=1$ for $k=1, \cdots, N$, then Spearman's $\rho$ is asymptotically equivalent to either of Kendall's $\tau$, product moment correlation 
coefficient and probability of concordance. Under Farlie's specification, however, LMPRT is given by

$$
S_{N}\left(\boldsymbol{R}_{N}\right)=\sum_{k=1}^{N}\left(1-\frac{2 R_{k}}{N+1}\right)\left(1-\frac{2 Q_{k}}{N+1}\right),
$$

which is equivalent to Spearman's $\rho$.

(d) Hájek's model.

Here we consider Hájek's model proposed in [8], p. 75. Let $\left(X_{k}, Y_{k}\right), k=1, \cdots$, $N$, be random variables defined by $X_{k}=X_{k}^{*}+c_{k} \Delta Z_{k}, Y_{k}=Y_{k}^{*}+c_{k} \Delta Z_{k}$, where $\left\{X_{k}^{*}\right\}$, $\left\{Y_{k}^{*}\right\}$ and $\left\{Z_{k}\right\}$ are mutually independent and each one is an i.i.d sequence, while the $c$ 's are known constants and $\Delta$ is an unknown parameter. Let $f, g$ and $M$ denote the density functions of $X^{*}$ and $Y^{*}$ and the distribution function of $Z$ respectively. Then the density function of $\left(X_{k}, Y_{k}\right)$ is given by

$$
h_{k}(x, y)=\int_{-\infty}^{\infty} f\left(x-c_{k} \Delta z\right) g\left(y-c_{k} \Delta z\right) d M(z) .
$$

We assume here that required assumtions hold and that the first and second differentiation with respect to $\Delta$ can be taken under the integral sign and also that $Z$ has a finite variance. Then we can get

$$
S_{N}\left(\boldsymbol{R}_{N}\right)=-E Z \sum_{k=1}^{N} c_{k}\left(E_{0}\left[\frac{f^{\prime}\left(X_{k}\right)}{f\left(X_{k}\right)} \mid R_{k}\right]+E_{0}\left[\frac{g^{\prime}\left(Y_{k}\right)}{g\left(Y_{k}\right)} \mid Q_{k}\right]\right) .
$$

If either $E Z=0$ or $c_{k}=1$ for any $k$, then $S_{N}\left(\boldsymbol{R}_{N}\right)$ is identically equal to zero, and hence useless. Now a straight-forward calculation leads to

$$
T_{N}\left(\boldsymbol{R}_{N}\right)=2(\operatorname{var} Z) \sum_{k=1}^{N} E_{0}\left[\frac{f^{\prime}\left(X_{k}\right)}{f\left(X_{k}\right)} \mid R_{k}\right] E_{0}\left[\frac{g^{\prime}\left(Y_{k}\right)}{g\left(Y_{k}\right)} \mid Q_{k}\right] .
$$

The statistic (4.8) is equivalent to that of [8], p. 76 , but the assumptions right here is stronger than those in [8].

\section{Asymptotic normality of the statistic $S_{N}\left(\boldsymbol{R}_{N}\right)$ under the null hypothesis.}

The exact distribution of the statistic $S_{N}\left(\boldsymbol{R}_{N}\right)$ is hard to obtain when the sample size $N$ is large, so that we show that when $\theta=0$ the limiting distribution is normal under some regularity conditions. First we need the following lemma which is a slight generalization of Lemma 6.1 of [6], but a variation of Lemma 2.1 of [11] as well, and which can be proved by using the martingale theory due to Doob [4] as in [11].

LEMMA 1. Let $\boldsymbol{X}_{N 1}, \cdots, \boldsymbol{X}_{N N}$ be an i.i.d sequence of p-variate random variables whose components are also independent. Let $\boldsymbol{R}_{N k}, k=1, \cdots, N$, be the rank vector of $\boldsymbol{X}_{N k}$ and $\phi$ be a Borel measurable function of p variables such that $E \psi^{2}(\boldsymbol{X})<\infty$. Then it holds that

$$
\lim _{N \rightarrow \infty} E\left[E\left(\phi\left(\boldsymbol{X}_{N_{1}}\right) \mid \boldsymbol{R}_{N_{1}}\right)-\phi\left(\boldsymbol{X}_{N 1}\right)\right]^{2}=0
$$

Next we define 


$$
T_{N}=N^{-1 / 2} \sum_{k=1}^{N} \sum_{r=1}^{q} c_{N k r} \lambda_{r} \psi_{r}\left(\boldsymbol{X}_{N k}\right)
$$

and

$$
\bar{c}_{N r}=N^{-1} \sum_{k=1}^{N} c_{N k r} \quad \text { for } r=1, \cdots, q .
$$

And we assume that

ASSUMPTION $\mathrm{A}_{6}$.

ASSUMPTION $\mathrm{A}_{7}$.

$$
\max _{k, 1} c^{2}{ }_{N k r}=0(1)
$$

$$
N^{1-2 p} \sum_{(\rightarrow) t} E_{0} \psi_{r}\left(X_{N\left(i_{1}\right) 1}, \cdots, X_{N(i p) p}\right) E_{0} \psi_{s}\left(X_{N\left(j_{1}\right) 1}, \cdots, X_{N(j p) p}\right)=\circ(1)
$$

for $t=1, \cdots, p$ and $r, s=1, \cdots, q$, where $X_{N(i) r}$ is the $i$-th order statistic among $X_{N 1 r}, \cdots, X_{N N r}$, and $\sum_{(\rightarrow) l}$ means the sum over all possible sets of ranks, $\left(i_{1}, \cdots, i_{p}\right)$ and $\left(j_{1}, \cdots, j_{p}\right)$, under the sole condition that $i_{t}=j_{t}$.

Verification of Assumption $A_{7}$ may happen to cause some difficulty. But $A_{7}$ is satisfied for Examples (a), (b) and (c) in Section 4 and the following lemma stated without proof gives a simple sufficient condition for $A_{7}$ to be satisfied.

LEMMA 2. If $\phi_{r}(\boldsymbol{x})=\sum_{t=1}^{n_{r}} h_{1 t r}\left(x_{1}\right) \cdots h_{p t r}\left(x_{p}\right)$ for $r=1, \cdots, p$ and if

$$
\sum_{k=1}^{N} E_{0}\left[h_{s t r}\left(X_{N k s}\right) \mid R_{N k s}\right]=0 \quad \text { for } s, r=1, \cdots, p \text { and } t=1, \cdots, n_{r}
$$

then $\left\{\psi_{r}\right\}_{r=1, \cdots, p}$ satisfies (5.5).

Asymptotic equivalence of $N^{-1 / 2} S_{N}\left(R_{N}\right)$ and $T_{N}$ is shown by the following lemma.

LEMma 3. Assume that Assumptions $A_{1}, A_{6}$ and $A_{7}$ are satisfied and also that $E_{0} \psi_{r}^{2}(\boldsymbol{X})<\infty$ for $r=1, \cdots, q$. Then $N^{-1 / 2} S_{N}\left(\boldsymbol{R}_{N}\right)-T_{N}$ converges to zero in probability as $N \rightarrow \infty$ under $H_{N 0}$.

Proof. We shall show that $E_{0}\left(N^{-1 / 2} S_{N}\left(\boldsymbol{R}_{N}\right)-T_{N}\right)^{2} \rightarrow 0$ as $N \rightarrow \infty$. Define

$$
Y_{N k r}=E_{0}\left[\psi_{r}\left(\boldsymbol{X}_{N k}\right) \mid \boldsymbol{R}_{N k}\right]-\phi_{r}\left(\boldsymbol{X}_{N k}\right) \text {. }
$$

Then

$$
\begin{aligned}
& E_{0}\left(N^{-1 / 2} S_{N}\left(\boldsymbol{R}_{N}\right)-T_{N}\right)^{2} \\
& =N^{-1} E_{0}\left(\sum_{k=1}^{N} \sum_{r=1}^{q} c_{N k r} \lambda_{r} Y_{N k r}\right)^{2} \\
& =N^{-1} \sum_{k=1}^{N} \sum_{r=1}^{q} \sum_{s=1}^{q} c_{N k r} c_{N k s} \lambda_{r} \lambda_{r} E_{0} Y_{N 1 r} Y_{N 1 s} \\
& +N^{-1} \sum_{k \neq k^{\prime}} \sum_{r=1}^{q} \sum_{s=1}^{q} c_{N k r} c_{N k^{\prime} s} \lambda_{r} \lambda_{s} E_{0} Y_{N 1 r} Y_{N 2 s} .
\end{aligned}
$$

In view of symmetricity of $Y_{N k r}$ with respect to the subscript $k$. By virtue of $\mathrm{A}_{6}$ and Lemma 1, the first term of (5.9) tends to zero as $N \rightarrow \infty$. Now we turn to the second term of (5.9). Since

$$
N^{-1} \sum_{k \neq k^{\prime}} c_{N k r} c_{N k^{\prime} s}=0(N),
$$


by Assumption $A_{6}$, we have only to show that

$$
E_{0}\left(Y_{N 1 r} Y_{N 2 s}\right)=0\left(N^{-1}\right) \text {. }
$$

Now $A_{1}$ implies that

$$
E_{0} \dot{\psi}_{r}(\boldsymbol{X})=0 \quad \text { for } r=1, \cdots, q,
$$

and hence

$$
\begin{aligned}
& E_{0}\left(Y_{N 1 r} Y_{N 2 s}\right) \\
& =E_{0}\left\{E_{0}\left(\psi_{r}\left(\boldsymbol{X}_{N 1}\right) \mid \boldsymbol{R}_{N_{1}}\right) E_{0}\left(\phi_{s}\left(\boldsymbol{X}_{N 2}\right) \mid \boldsymbol{R}_{N 2}\right)-\psi_{r}\left(\boldsymbol{X}_{N 1}\right) E_{0}\left(\psi_{s}\left(\boldsymbol{X}_{N 2}\right) \mid \boldsymbol{R}_{N 2}\right)\right. \\
& \left.-\psi_{s}\left(\boldsymbol{X}_{N 2}\right) E_{0}\left(\phi_{r}\left(\boldsymbol{X}_{N 1}\right) \mid \boldsymbol{R}_{N 1}\right)\right\} .
\end{aligned}
$$

Considering the rank conditional expectation, we can easily get

$$
\begin{aligned}
& E_{0}\left(Y_{N 1 r} Y_{N 2 s}\right) \\
& =-E_{0}\left(E_{0}\left(\psi_{r}\left(\boldsymbol{X}_{N 1}\right) \mid \boldsymbol{R}_{N 1}\right) E_{0}\left(\phi_{s}\left(\boldsymbol{X}_{N 2}\right) \mid \boldsymbol{R}_{N 2}\right)\right) \\
& =-(N(N-1))^{-p} \sum_{i_{1} \neq j_{1}, \cdots, i_{p} \neq j_{p}} E_{0} \phi_{r}\left(X_{N\left(i_{1}\right) 1}, \cdots, X_{N\left(i_{p}\right) p}\right) E_{0} \psi_{s}\left(X_{N\left(j_{1}\right) 1}, \cdots, X_{N\left(j_{p}\right) p}\right) \\
& =-(N(N-1))^{-p}\left\{\sum_{i_{1} j_{1}} \cdots \sum_{i_{p} j_{p}}-\sum_{t=1}^{p} \sum_{(\cdot) t}+B_{2} \sum_{t_{1} \neq t_{2}} \sum_{\substack{i_{t_{1}}=\sum_{t_{1}} \\
i_{t_{2}}=j_{t_{2}}}}+\cdots+B_{i_{i_{1}}=j_{1}} \sum_{i_{p}=j_{p}}\right\} \\
& \times E_{0} \psi_{r}\left(X_{N\left(i_{1}\right) 1}, \cdots, X_{N\left(i_{p}\right) p}\right) E_{0} \psi_{s}\left(X_{N\left(j_{1}\right) 1}, \cdots, X_{N\left(j_{p}\right) p}\right),
\end{aligned}
$$

where $B_{2}, \cdots, B_{p}$ are constants depending only on $p$ and each summation extends over all possible values of ranks subject to the specified conditions. Using (5.12) and $A_{7}$, it can be easily shown that (5.11) holds. This completes the proof.

Using above lemmas, we can get the following Theorem.

Theorem 3. Assume that Assumptions $A_{1}, A_{6}$ and $A_{7}$ are satisfied and $E_{0} \psi_{r}{ }^{2}(X)$ exists for $r=1, \cdots, q$. Then, under $H_{N 0}, N^{-1 / 2} S_{N}\left(\boldsymbol{R}_{N}\right)$ is asymptotically normal with mean 0 and variance $\sigma^{2}{ }_{N}$ as $N \rightarrow \infty$, where

$$
\sigma^{2}{ }_{N}=N^{-1} \sum_{k=1}^{N} \sum_{r=1}^{q} \sum_{s=1}^{q} c_{N k r} c_{N k s} \lambda_{r} \lambda_{s} E_{0} \psi_{r}(\boldsymbol{X}) \psi_{s}(\boldsymbol{X})
$$

Proof. By virtue of Lemma 3, it sufficies to show that $T_{N}$ has the asserted asymptotic distribution. It can be easily shown that $E_{0} T_{N}=0$ and $\operatorname{Var}_{0} T_{N}=\sigma^{2}{ }_{N}$. If $\sigma^{2}{ }_{N} \rightarrow 0$ as $N \rightarrow \infty$, then $T_{N}$ is asymptotically degenerate normal. Now suppose $\sigma^{2}{ }_{N} \rightarrow M>0$ as $N \rightarrow \infty$ and put

$$
T_{N r}=N^{-1 / 2} \sum_{k=1}^{N} c_{N k r} \lambda_{r} \psi_{r}\left(\boldsymbol{X}_{N k}\right),
$$

then it holds that

$$
T_{N r} \sim N\left(0, N^{-1} \sum_{k=1}^{N} c^{2}{ }_{N k r} \lambda^{2}{ }_{r} E_{0} \psi_{r}^{2}(\boldsymbol{X})\right)
$$

(see [8], Theorem V. 1.2). Using the method used in [8], p. 218, we can easily verify the Lindeberg condition and get the desired result. 


\section{Asymptotic normality of the statistic $S_{N}\left(\boldsymbol{R}_{N}\right)$ under local alternatives.}

In this section we investigate the limiting distribution of $N^{-1 / 2} S_{N}\left(\boldsymbol{R}_{N}\right)$ under local alternatives, using the notion of contiguity due to LeCam [10] and developed by Hájek [7] and in particular LeCam's lemmas stated elegantly in [8]. Let $\theta^{0}=$ $\left(\theta_{1}^{0}, \cdots, \theta_{q}^{0}\right)$ be fixed numbers such that $\sum_{r=1}^{q} \theta_{r}^{0}>0$ and $\theta_{r}^{0}\left(\sum_{i=1}^{q} \theta_{i}^{0}\right)^{-1}=\lambda_{r}$ for $r=1, \cdots, q$. Alternative hypothesis to be considered is that $\boldsymbol{X}_{N i}$ has a density function $f\left(\boldsymbol{x}, N^{-1 / 2} \boldsymbol{c}_{N i} \theta^{0}\right)$ independently for $i=1, \cdots, N$. We use the same analysis as in [1], $[7],[8]$ and [11], and so necessary statistics and quantities are given here.

$$
\begin{gathered}
V_{N k}=f\left(\boldsymbol{X}_{N k}, N^{-1 / 2} \boldsymbol{c}_{N k} \theta^{0}\right) / f\left(\boldsymbol{X}_{N k}\right), \quad k=1, \cdots, N, \\
L_{N \theta^{0}}=\log \left(\prod_{k=1}^{N} V_{N k}\right), \\
W_{N}=2 \sum_{k=1}^{N}\left(V_{N k}^{1 / 3}-1\right), \\
\widetilde{T}_{N}=N^{-1 / 2} \sum_{k=1}^{N} \sum_{r=1}^{q} c_{N k r} \theta_{r}^{0} \frac{\partial}{\partial \theta_{r}} f\left(\boldsymbol{X}_{N k}, \boldsymbol{0}\right) / f\left(\boldsymbol{X}_{N k}\right), \\
b^{2}=\lim _{N \rightarrow \infty} N^{-1} \sum_{k=1}^{N} \sum_{r=1}^{q} \sum_{s=1}^{q} c_{N k r} c_{N k s} \theta_{r}^{0} \theta_{s}^{0} E_{0} \phi_{r}(\boldsymbol{X}) \phi_{s}(\boldsymbol{X}) .
\end{gathered}
$$

We give a lemma needed to establish the asymptotic distribution of $N^{-1 / 2} S_{N}\left(\boldsymbol{R}_{N}\right)$ without proof because it is similar to that stated in [8], VI. 2.1.

LemMa 4. If $E_{0} \psi_{r}^{2}(\boldsymbol{X})$ exists for every $r$ and Assumptions $A_{1}$ and $A_{6}$ hold, then for any $\varepsilon>0$,

and $\left(T_{N}, \widetilde{T}_{N}-\frac{1}{2} b^{2}\right)$ is asymptotically bivariate normal under $H_{N 0}$.

Lemma 4 implies that $W_{N}$ is asymptotically normal under $H_{N 0}$ and that the alternative hypothesis considered is contiguous to $H_{N 0}$, so that we can apply LeCam's lemma.

THEOREM 4. If $E_{0} \psi_{r}^{2}(X)$ exists for every $r$ and the Assumption $A_{1}, A_{6}$ and $A_{7}$ are satisfied, then the asymptotic distribution of $N^{-1 / 2} S_{N}\left(\boldsymbol{R}_{N}\right)$ under the alternative sequence $\theta_{N}=N^{-1 / 2} \theta^{0}$ is normal with mean $\left(\sum_{r=1}^{q} \theta_{r}^{0}\right)^{-1} b^{2}$ and variance $\left(\sum_{r=1}^{q} \theta_{r}^{0}\right)^{-2} b^{2}$.

PROOF. By LeCam's third lemma in [8] and Lemma 4, asymptotic equivalence of $\left(N^{-1 / 2} S_{N}\left(\boldsymbol{R}_{N}\right), L_{N 0}\right)$ and $\left(T_{N}, \widetilde{T}_{N}-\frac{1}{2} b^{2}\right)$ implies that we may calculate $\operatorname{Cov}_{0}\left(T_{N}\right.$, $\left.\widetilde{T}_{N}-\frac{1}{2} b^{2}\right)=E_{0} T_{N} \tilde{T}_{N}$ to get the asymptotic mean. Now 


$$
\begin{aligned}
E_{0} T_{N} \tilde{T}_{N} & =E_{0} N^{-1}\left(\sum_{r=1}^{q} \theta_{r}^{0}\right)^{-1} \sum_{k, k^{\prime}} \sum_{r=1}^{q} \sum_{s=1}^{q} c_{N k r} c_{N k^{\prime} s} \theta_{r}^{0} \theta_{s}^{0} \psi_{r}\left(\boldsymbol{X}_{N k}\right) \psi_{s}\left(\boldsymbol{X}_{N k^{\prime}}\right) \\
& =N^{-1}\left(\sum_{r=1}^{q} \theta_{r}^{0}\right)^{-1} \sum_{k=1}^{N} \sum_{r=1}^{q} \sum_{s=1}^{q} c_{N k r} c_{N k s} \theta_{r}^{0} \theta_{s}^{0} E_{0} \psi_{r}(\boldsymbol{X}) \psi_{s}(\boldsymbol{X}) \\
& \rightarrow\left(\sum_{r=1}^{q} \theta_{r}^{0}\right)^{-1} b^{2} \quad \text { as } N \rightarrow \infty
\end{aligned}
$$

The asymptotic variance may be obtained under the null hypothesis and hence it is expressed as given in the statement of the theorem. This completes the proof with the aid of LeCam's third lemma.

\section{Acknowledgement}

I should like to thank Professor M. Okamoto for his continued guidance and Dr. Y. Fujikoshi and Mrs. M. Yamaguchi for their many helpful discussions and advices.

\section{References}

[1] J. N. Adichie, Asymptotic efficiency of a class of non-parametric tests for regression parameters, Ann. Math. Statist., 38 (1967), 884-893.

[2] T.W. ANDERson, An Introduction to Multivariate Statistical Analysis, John Wiley (1957), New York.

[3] S. Bhuchongkul, A class of non-parametric tests for independence in bivariate populations, Ann. Math. Statist., 35 (1964), 138-149.

[4] J.L. Doob, Stochastic Processes, John Wiley (1953), New York.

[5] D.F.G. FARLIE, The performance of some correlation coefficients for a general bivariate distribution, Biometrika., 47 (1960), 307-323.

[6] J. HÁJEK, Some extentions of the Wald-Wolfowitz-Noether theorem, Ann. Math. Statist., 32 (1961), 506-523.

[7] J. HÁJeK, Asymptotically most powerful rank tests, Ann. Math. Statist., 32 (1962), 11241147.

[8] J. HÁJEK and Z. S̆IDÁK, Theory of Rank Tests, Academia (1967), Prague.

[9] H.S. KonIJN, On the power of certain tests for independence in bivariate populations, Ann. Math. Statist., 27 (1956), 300-323.

[10] L. LECAM, Locally asymptotically normal families of distribution, Univ. of Calif. Publs. Statist., 3 (1960), 37-98.

[11] T.K. MATThes and D. R. TRUAX, Optimal invariant rank tests for the k-sample problem, Ann. Math. Statist., 36 (1965), 1207-1222.

[12] M. L. Puri and P.K. Sen, Nonparametric Methods in Multivariate Analysis, John Wiley (1971), New York. 